Größe von $f_{\mathrm{r}}$. Ferner ergibt sich aus Whiffens Messungen an Campher in verschiedenen Lösungsmitteln, die auch in Tab. 5 aufgeführt sind, ebenfalls eine Folge von Werten von $f_{\mathrm{r}}$, die zwar im Absolutbetrag kleiner sind, jedoch etwa parallel denen in Abb. 7 verlaufen. Berücksichtigt man aber den Umstand, daß die Camphermolekeln eine innere freie Drehbarkeit besitzen, und daß daraus ein kleinerer effektiver Molekelradius resultiert, so verschieben sich die Campherwerte im erwarteten Sinne. Wir gehen hier nicht weiter auf die entsprechende Diskussion der übrigen experimentellen Untersuchungen ein. Sie soll in einer anderen Arbeit nachgeholt werden.

Bei dieser Ermittlung des empirischen Mikroreibungsfaktors der Rotation mit Hilfe der Dipolorientierung ist folgende Schwierigkeit zu bedenken. Das elektrische Moment des Gelösten hat zur Folge, daß zwischen dem Gelösten und den umgebenden Lösungsmittelmolekeln andere Kräfte wirken, als zwischen den unpolaren Lösungsmittelmolekeln selbst. Erforderlich wäre u. a. eine Reduktion der empirischen Daten auf das Moment Null, die im Augenblick nicht möglich erscheint. Aus der Tab. 5 kann man jedoch Hinweise auf Einflüsse des Momentes entnehmen. Und zwar scheint es, da $\beta$ bei festen $r / r_{\mathrm{L}}$ dann $f_{\mathrm{r}}$ besonders groß ist, wenn das Moment groß ist. Doch möchten wir dieser Feststellung kein besonderes Gewicht beilegen. Da mit $r / r_{\mathrm{L}}$ auch die Momente der Whiffenschen Stoffe im Mittel zunehmen, würde eine Reduktion auf gleiche Mo- mente den Anstieg von $f_{\mathrm{r}}$ mit $r / r_{\mathrm{L}}$ in Abb. 7 eher abschwächen. In Tab. 5 sind ferner die schwach polaren reinen Flüssigkeiten $p$-Xylol, Toluol und $\alpha$ Bromnaphthalin aufgeführt. Hier wurde, z. Tl. ebenfalls von Whiffen, die Relaxation dieser schwachen Dipole in der eigenen praktisch nicht assoziierenden Flüssigkeit gemessen. Es ist also völlige „Milieugleichheit" hinsichtlich der Kräfte zwischen Gelöstem und Lösungsmittel und im Lösungsmittel selbst vorhanden, und wegen der sehr kleinen Momente treten die Störungen nicht auf, die üblicherweise in konzentrierten polaren Lösungen bzw. reinen polaren Flüssigkeiten zu berücksichtigen sind. Die sich ergebenden Mikroreibungsfaktoren $f_{\mathrm{r}}$ passen sehr gut zu den übrigen Whiffenschen Werten in verdünnten Lösungen. Daraus folgt, daß schwache Momente in verdünnter Lösung offenbar keine starke Störung darstellen. Insgesamt schließen wir deshalb, daß die permanenten Momente bei der Diskussion der Radienabhängigkeit zwar im Auge behalten werden müssen, jedoch die Größenordnung der Mikroreibungsfaktoren in unseren Beispielen wohl nicht beeinflussen. Eine genauere Ermittlung der Radienabhängigkeit von $f_{\mathrm{r}}$ würde die Untersuchung eines größeren Intervalls von $r / r_{\mathrm{L}}$ als bei den bisher vorliegenden vergleichbaren Untersuchungen zur Voraussetzung haben, doch scheint es, daß auch hier $f_{\mathrm{r}}$ mit $r / r_{\mathrm{L}}$ ansteigt.

Herrn Dr. A. Gierer danken wir für wertvolle Ratschläge und Diskussionen.

\title{
Molekulare Theorie der Mikroreibung
}

\author{
Von A. GIERER und K. WIRTz
}

\author{
Aus dem Max-Planck-Institut für Physik, Göttingen \\ (Z. Naturforschg. 8a, 532-538 [1953]; eingegangen am 25. Juni 1953) \\ Clemens Schaefer zum 75. Geburtstag gewidmet
}

\begin{abstract}
Die Flüssigkeitsschichten, die um ein sich bewegendes Molekül herumfließen, haben infolge der endlichen Abmessungen der Flüssigkeitsmolekeln eine endliche Dicke. Die Berücksichtigung dieses Umstandes führt zu einer Modifikation der Stokesschen Gesetze der Kontinuumstheorie für den Zusammenhang zwischen Reibung und Viskosität. Es ergibt sich die richtige Größenordnung und ungefähr die richtige Radienabhängigkeit der beobachteten Mikroreibung.
\end{abstract}

1. Makroskopische und mikroskopische Reibung

$\mathrm{E}$ ine Flüssigkeit befinde sich zwischen zwei gegeneinander mit der Geschwindigkeit $v$ bewegten Wänden vom Abstand $L$. Zur Aufrechterhaltung der Geschwindigkeit gegen die Reibung der Flüssigkeit muß auf die Wände die Kraft $K$ wirken, die durch die folgende Beziehung gegeben ist:

$$
K=\eta F \frac{v}{L}=\eta F \frac{\mathrm{d} v}{\mathrm{~d} z}
$$


( $\eta=$ Viskosität der Flüssigkeit; $\boldsymbol{F}=$ Fläche der bewegten Wände; $\mathrm{d} v / \mathrm{d} z=$ Geschwindigkeitsgradient senkrecht zu den Wänden). Die Aufgabe bestehe darin, diese Beziehung der Kontinuumstheorie, die die Newtonsche Definition der Viskositätskonstanten $\eta$ darstellt, für Bereiche von molekularer GröBenordnung neu zu formulieren. A priori muß man darauf gefaßt sein, an Stelle der makroskopischen Größen $v, \mathrm{~d} v / \mathrm{d} z$ und $F$ modifizierte molekulare Ausdrücke zu finden.

Die für das vorliegende Problem wichtigste Modifikation, die den Hauptteil der empirischen Mikroreibung liefern wird, besteht in der Berücksichtigung der Tatsache, daß sich in der nahgeordneten Flüssigkeit nur Schichten mit der endlichen Dicke $l$ $(l \approx$ Moleküldurchmesser) gegeneinander verschieben können. Infolgedessen tritt an die Stelle des Differentialquotienten $\mathrm{d} v / \mathrm{d} z$ in ( 1 ) der Differenzenquotient $\Delta v / l$, wo $\Delta v$ der Geschwindigkeitsunterschied zweier benachbarter Molekülschichten ist. An Stelle von (1) tritt also

$$
K=\eta \boldsymbol{F} \Delta v / l .
$$

Hinsichtlich des Verhaltens der wirksamen Fläche $\boldsymbol{F}$ beim Übergang zu molekularen Dimensionen kann man folgende grobe Überlegung anstellen. Für eine makroskopische Kugel vom Radius $r$ ist $F=4 \pi r^{2}$. Für ein Molekül erschiene es zunächst sinnvoller, anstatt von wirksamer Oberfläche von der Zahl der Bindungen zu nächsten Nachbarn zu sprechen. Man kann aber leicht überschlagen, daß kein großer Fehler entsteht, auch beim Molekül $F=4 \pi r^{2}$ zu setzen. Z. B. nimmt für $r=r_{\mathrm{L}}\left(r_{\mathrm{L}}=\right.$ Radius der Moleküle der umgebenden Flüssigkeit) in dichtester Kugelpackung ein Molekül in einer hexagonalen Schicht die Fläche $\Delta F=3 r^{2}$ (Sechseck mit der Kantenlänge $r$ ) ein. Auf dieser Fläche wirkt die Schubkraft der darüber liegenden Schicht auf das Molekül. In dieser Nachbarschicht berührt das Molekül drei Nachbarn (entsprechend drei Bindungen). Also entfällt auf einen der insgesamt 12 Nachbarn die effektive Oberfläche $r^{2}$. Hätte man die Oberfläche $F=4 \pi r^{2}$ angesetzt, so hätte sich pro Nachbar $(4 \pi / 12) r^{2} \approx r^{2}$ ergeben, d. h. praktisch derselbe Wert. In den meisten Flüssigkeiten dürfte die molekulare Koordination angenähert einer dichtesten Kugelpackung entsprechen. Für andere Koordinationsverhältnisse dürfte sich ein ähnliches Resultat ergeben. Man kann also die Oberfläche $F$ eines Moleküls mit dem ,makroskopischen“ Wert $4 \pi r^{2}$ ansetzen.
Daß man schließlich auch im molekularen Bereich mit dem Mittelwert $v$ der Geschwindigkeit operieren darf, wird in Abschn. 2 durch eine kinetische Betrachtung erläutert werden.

Für eine Kugel ist nach Stokes die Reibungskraft der Translation:

$$
K=6 \pi \eta r v
$$

und das Reibungsmoment der Rotation mit der Winkelgeschwindigkeit $\varphi$ :

$$
M=8 \pi \eta r^{3} \dot{\varphi} .
$$

Unser Ziel ist, für eine Kugel von molekularer Dimension die Modifikationen dieser Gesetze zu formulieren, die von der endlichen Dicke der die Kugel umgebenden Molekelschichten herrühren. Drückt man wie in der vorangehenden Arbeit $^{1}$ diese Modifikationen durch korrigierende ,Mikroreibungsfaktoren" $f_{\mathrm{t}}$ bzw. $f_{\mathrm{r}}$ in den Stokesschen Gesetzen aus:

$$
K=6 \pi \eta r f_{\mathrm{t}} v, M=8 \pi \eta r^{3} f_{\mathrm{r}} \dot{\varphi},
$$

so besteht unsere Aufgabe in der Berechnung dieser Faktoren.

\section{Kinetische Formulierung der Viskositätsgleichung}

Die Platzwechseltheorie führt den viskosen Fluß auf thermisch aktivierte Einzelschritte der Molekeln zurück. Im idealen Kristallgitter verursacht eine Schubkraft keine von Null verschiedene Kraftkomponente auf das einzelne Molekül, so daß keine Vorzugsrichtung etwaiger Platzwechsel verursacht wird. Viskoser Fluß tritt also im idealen Kristall nicht auf. In der Flüssigkeit ist die Fernordnung durch einzelne Störungen, Löcher genannt, unterbrochen. Es gehört offenbar zum Wesen der gestörten Flüssigkeitsstruktur, daß die Komponente der Schubkraft auf das einzelne Teilchen im Mittel von Null verschieden ist, so daß eine Bewegung in einer Vorzugsrichtung möglich wird. Da die Einzelprozesse als statistisch unabhängig angesehen werden, ergibt sich die mittlere Verschiebung von Molekelschichten beim viskosen Fluß als resultierend aus vielen bevorzugten Platzwechseln der Moleküle einer Schicht gegenüber den Nachbarschichten.

Es ist schwierig, sich ein genaueres Bild von den Platzwechseln des viskosen Flusses und den sie ermöglichenden Störungen der Fernordnung zu machen. Ein Beispiel einer solchen Störung wäre ein Molekül, dem ein Nachbar fehlt. Ein Platzwechsel des viskosen Pro-

1 A. Spernol u. K. Wirtz, Z. Naturforschg. 8 a, 522 [1953]; voranstehend. 
zesses betrifft wahrscheinlich nur die Lösung der Bindung zu einem kleinen Bruchteil der nächsten Nachbarn. Damit sich ein Teilchen relativ zu allen seinen Nachbarn bewegt, sind mehrere Platzwechsel vom viskosen Typ notwendig. Die Experimente sprechen dafür, daß in der Regel nur ein Typ derartiger Platzwechsel vorherrscht, und daß in der normalen Flüssigkeit Diffusion und Rotation überwiegend durch sie vermittelt werden.

Wirkt auf eine Flüssigkeitsschicht die Schubkraft pro Flächeneinheit $K / F$, also von den darüber liegenden Molekülen auf die Fläche $\Delta F$ eines Moleküls die Kraft $(K / F) \Delta F$, und erfolgen $j$ Platzwechsel pro Sekunde und Molekül um eine Strecke $d$, so folgt für die mittlere Relativgeschwindigkeit $\Delta v$ der Schichten ${ }^{2}$

$$
\Delta v=\frac{K}{F} \Delta F \frac{\varkappa k T}{j d^{2}} .
$$

Hier ist $\varkappa$ ein von der Nahordnung abhängiger Faktor, der für das Folgende keine Rolle spielt. Mit dieser Geschwindigkeit verschieben sich alle Schichten gegeneinander. Ist $l$ der mittlere Abstand der Schichten, so ist das Geschwindigkeitsgefälle

$$
\frac{\Delta v}{l}=\frac{K}{F} \frac{1}{\eta}=\frac{K}{F} \Delta F \frac{j d^{2}}{\varkappa k T l} .
$$

Mit $\Delta F=l^{2}$ und $d=l$ erhält man aus (7) die oft gebrauchte Näherung

$$
\eta=\frac{\varkappa k T}{j l^{3}} .
$$

Bei Molekülen tritt die Gl. (7) an die Stelle von (1). $\mathrm{d} v / \mathrm{d} z$ ist durch $\Delta v / l$ ersetzt. (7) ist die primäre Viskositätsgleichung, die erst im makroskopischen Fall in die differentielle Beziehung (1) der Kontinuumstheorie übergeht. Die mittlere Geschwindigkeit $v$ bleibt von diesem Übergang unbeeinflußt. Es bestätigt sich ferner, daß bei der molekularen Reibung das Auftreten endlicher Schichtdicken $l$ in der Umgebung des wandernden Moleküls berücksichtigt werden muß. Das Modell legt außerdem nahe, die Kräfte, die zur Aufrechterhaltung der mittleren Geschwindigkeit $v$ eines Moleküls an einzelnen Teilen seiner Oberfläche angreifen, einfach zu addieren, wie es bei der Platzwechseltheorie der Viskosität mit den Kräften auf die einzelnen Flächenelemente der Schichtenebene geschieht.

Bei der Untersuchung der Reibung der Translation oder Rotation einer molekularen Kugel unter diesen Gesichtspunkten muß allerdings außerdem

${ }^{2}$ Vgl. z. B. K. Wirtz, Z. Naturforschg. 3a, 672 [1948]. beachtet werden, daß es Arten von molekularen Platzwechseln gibt, die nichts zum viskosen Fluß, wohl aber zur Teilchenbewegung beitragen. Platzwechsel eines Teilchens z. B. relativ zu allen seinen Nachbarn, wie sie sowohl im Kristall als auch in der Flüssigkeit vorkommen können, haben mit einer Verschiebung von Schichten gegeneinander nichts zu tun, tragen aber zur Diffusion bei. Ebenso hat die aktivierte spontane Drehung eines Moleküls um eine Achse aus einer Gleichgewichtslage in eine andere, wenn sie ohne Vermittlung von Löchern verläuft, nichts mit der viskosen Rotation zu tun. Man kann nicht a priori erwarten, daß sich derartige Prozesse in einfacher Weise mit den Platzwechseln des viskosen Flusses korrelieren lassen. Vielmehr kommt der Wanderungsanteil, der auf derartigen ,selbständigen“" Platzwechseln beruht, additiv zu demjenigen hinzu, der mit der viskosen Verschiebung von Schichten zusammenhängt (vgl. auch Abschn. 6).

Aus den Überlegungen der beiden ersten Abschnitte ergibt sich, daß der Übergang von der Gl. (1) der Kontinuumstheorie zu der für molekulare Dimensionen gültigen Beziehung

$$
K=\eta \sum \Delta F \frac{\Delta v}{l}
$$

gerechtfertigt ist, wenn

1. selbständige Platzwechsel selten sind, und

2. zwischen dem betrachteten Teilchen und denen des Lösungsmittels annähernd die gleichen Kräfte wirken, wie zwischen denen des Lösungsmittels selbst.

Die zweite Bedingung entspricht der Voraussetzung von Stokes, daß der bewegte Körper von einer festhaftenden Flüssigkeitsschicht bedeckt sein soll, die bewirkt, daß der Reibungsvorgang sich nur in der Flüssigkeit abspielt.

\section{Mikroreibung der Rotation}

Wir betrachten zunächst den einfacheren Fall der Rotation. Eine Kugel vom Radius $r$ (Abb. 1) rotiere unter der Einwirkung des Drehmoments $\boldsymbol{M}$ um die horizontale Achse mit der Winkelgeschwindigkeit $\dot{\varphi}$, Das hydrodynamische Umströmungsbild ist einfach und läßt sich als differentielle Rotation konzentrischer Kugelschalen auffassen. Hieran ändert auch die Berücksichtigung endlicher molekularer Schichtdicken $l$ nichts. Zwischen je zwei Ku- 
gelschalen wirkt insgesamt das Moment $M$. Ist der Radius der $m$-ten Schicht

$$
R_{m}=r+m l,
$$

so wirkt auf das Flächenelement $\mathrm{d} F_{m}$ der $m$-ten Schicht das Drehmoment

$$
\mathrm{d} M=\mathrm{d} K_{m} \cdot R_{m} \sin \vartheta .
$$

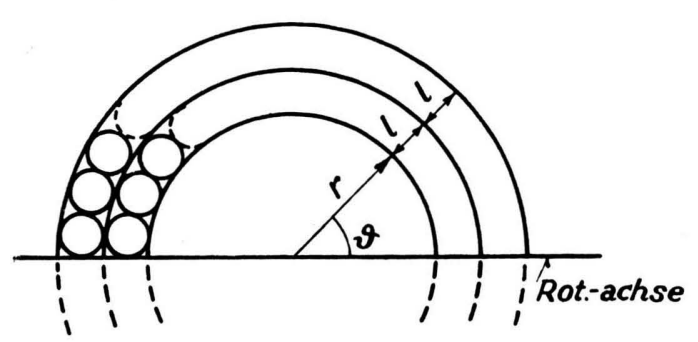

Abb. 1 .

Der Geschwindigkeitsunterschied zwischen zwei benachbarten Flächenelementen zweier Schichten ist, wenn $\dot{\varphi}_{m}$ die Winkelgeschwindigkeit der $m$-Schicht relativ zur Nachbarschicht ist

$$
\Delta v=R_{m} \sin \vartheta \cdot \dot{\varphi}_{m} .
$$

Dies gibt zusammen mit (9)

$$
\mathrm{d} M=\mathrm{d} F_{m} \frac{R_{m}^{2} \sin ^{2} \vartheta}{l} \eta \dot{\varphi}_{m} .
$$

Integriert über die Oberfläche der Schicht ergibt dies für das gesamte Moment:

$$
M=\frac{2}{3} \frac{4 \pi R_{m}^{4}}{l} \eta \dot{\varphi}_{m} .
$$

Diese Gleichung liefert den Zusammenhang zwischen dem wirkenden Moment $M$ und $\operatorname{der} \operatorname{durch} M$ verursachten Winkelgeschwindigkeit $\dot{\varphi}_{m}$ der $m$-ten Schicht. Die Summation über alle Schichten liefert die gesuchte totale Winkelgeschwindigkeit

$$
\dot{\varphi}=\sum_{m=0}^{\infty} \dot{\varphi}_{m}=\frac{3 M}{8 \pi \eta} \sum_{m=0}^{\infty} \frac{l}{R_{m}^{4}} .
$$

Ist $r \gg l$, so kann man die Summe in ein Integral verwandeln und erhält die Gleichung

$$
\frac{M}{\dot{\varphi}}=\varrho_{\mathrm{r}}{ }^{(\mathrm{makro})}=8 \pi \eta r^{3}
$$

also, wie es sein muß, die Stokessche Reibungsformel (4). Ist jedoch $r \approx r_{\mathrm{L}}$, so muß die Summe ausgewertet werden. In diesem Fall ist das erste Glied wesentlich größer als alle anderen. Man erhält an Stelle von (15), wenn man noch $l=2 r_{\mathrm{L}}$ setzt

$$
\dot{\varphi}=\frac{3 M}{8 \pi \eta}\left(\frac{2 r_{\mathrm{L}}}{r^{4}}+\sum_{m=1}^{\infty} \frac{2 r_{\mathrm{L}}}{R_{m}^{4}}\right) .
$$

Damit wird

$\frac{M}{\dot{\varphi}}=\varrho_{\mathrm{r}}{ }^{\text {(mikro) }}=\frac{8 \pi \eta}{3}\left(\frac{2 r_{\mathrm{L}}}{r^{4}}+\sum_{m=1}^{\infty} \frac{2 r_{\mathrm{L}}}{R_{m}^{4}}\right)^{-1}$.

Nähert man die Summe durch ein Integral an, so liefert sie den Wert

$$
\left[3 r^{3}\left(1+\frac{r_{\mathrm{L}}}{r}\right)^{3}\right]^{-1} .
$$

Vernachlässigt man dieses Glied für $r \approx r_{\mathrm{L}}$, so wird

$$
\varrho_{\mathrm{r}}^{(\mathrm{mikro})}=8 \pi \eta r^{3} \frac{1}{6} \frac{r}{r_{\mathrm{L}}}
$$

und man erhält für den in (5) definierten Mikroreibungsfaktor angenähert

$$
f_{\mathrm{r}}^{\prime}=\frac{\varrho_{\mathrm{r}}^{\text {(mikro) }}}{\varrho_{\mathrm{r}}^{\text {(makro) }}}=\frac{1}{6} \frac{r_{\mathrm{L}}}{r} .
$$

Nimmt man die Summe mit, so wird genauer

$$
f_{\mathrm{r}}^{\prime}=\left(6 \frac{r_{L}}{r}+\frac{1}{\left(1+r_{\mathrm{L}} / r\right)^{3}}\right)^{-1}
$$

$f_{\mathrm{r}}^{\prime}$ kann sich von dem empirischen $f_{\mathrm{r}}$ noch dadurch unterscheiden, daß zwischen dem Gelösten und dem Lösungsmittel andere Kräfte als im Lösungsmittel selbst herrschen, so wie durch die Effekte etwaiger ,,selbständiger Platzwechsel“" (Abschn. 2).

\section{Mikroreibung der Translation}

Während man im Falle der Rotation die Struktur des Mediums dadurch berücksichtigen konnte, daß man die Integration über den Geschwindigkeitsgradienten durch die Summation über Schichten molekularer Dicke ersetzte, läßt sich dieses Verfahren bei dem komplizierten Umströmungsbild der Translation nicht streng durchführen.

Jedenfalls soll sich für große Radien im Grenzfall die Stokesche Gleichung

$$
v=\frac{K}{6 \pi \eta r}
$$

ergeben. Auch diese Bewegung muß sich als Relativbewegung von Schichten von molekularer Dicke $2 r_{\mathrm{L}}$ darstellen lassen. Dies werde in einer ersten Näherung dadurch ausgedrückt, daß der Stokessche Ausdruck als Ergebnis einer Integration über derartige Schichten aufgefaßt wird. Dieses Integral kann man in eine Summe verwandeln:

$$
v \rightarrow \int \frac{K d r}{6 \pi \eta r^{2}} \rightarrow \sum \Delta v=\sum_{m=0}^{\infty} \frac{K}{6 \pi \eta} \frac{2 r_{\mathrm{L}}}{\left(r+2 m r_{\mathrm{L}}\right)^{2}} .
$$


Damit ergibt sich im Grenzfall die Stokessche Formel (22). In Wirklichkeit gibt diese Gleichung das hydrodynamische Umströmungsbild - im Gegensatz zur Rotation - nicht richtig wieder, da der radiale Geschwindigkeitsgradient senkrecht zur Translationsrichtung im hydrodynamischen Bild noch ein Glied mit $R_{m}^{-4}$ enthält, das in Gl. (23) nicht auftritt. Dennoch darf man erwarten, daß Gl. (23) die Relativgeschwindigkeiten der Schichten in 1. Näherung darstellt, so daß nun der zweite Schritt, die Abspaltung des ersten Gliedes und die Verwandlung des Rests in ein Integral, getan werden kann. Man erhält

$$
v=\frac{K}{6 \pi \eta r}\left(2 \frac{r_{\mathrm{L}}}{r}+\frac{1}{1+r_{\mathrm{L}} / r}\right)^{-1}
$$

und somit für den Mikroreibungsfaktor

$$
f_{\mathrm{t}}^{\prime}=\left(2 \frac{r_{\mathrm{L}}}{r}+\frac{1}{1+r_{\mathrm{L}} / r}\right)^{-1} \text {. }
$$

Eine genauere Betrachtung hätte die richtige Umströmung zu berücksichtigen. Die Gleichung

$$
\Delta v=\frac{K 2 r_{\mathrm{L}}}{\eta F}
$$

ist für gekrümmte Flächen um einen Faktor $c$ zu erweitern, der den mittleren Einfluß der Winkel zwischen Fläche, Kraft und Geschwindigkeitsgradient berücksichtigt. Für die $m$-te Schicht gilt

$$
\Delta v_{m}=\frac{K 2 r_{\mathrm{L}}}{\eta 4 \pi\left(r+2 m r_{\mathrm{L}}\right)^{2}} c_{m} .
$$

Die Gl. (23) besagt, daß bei dieser Näherung alle $c_{m}$ einheitlich $2 / 3$ gesetzt sind, um den Übergang zum Stokeschen Gesetz zu erhalten.

Man kann nun zur Prüfung dieser Näherung noch eine molekulare Betrachtung für die Relativbewegung zur ersten Schicht im Falle $r=r_{\mathrm{L}}$ anstellen. Ist die Relativgeschwindigkeit $\Delta v_{0}$ (z. B. in Richtung der Rotationsachse in Abb. 1), so müssen sich an der Oberfläche die Moleküle, die die Kugel an einer Stelle mit dem Winkel $\vartheta$ gegen die Translationsrichtung berühren, mit der Tangentialgeschwindigkeit $\Delta v_{0} / \sin \vartheta$ längs der Kugeloberfläche bewegen; die Kraft muß also ebenfalls eine Tangentialkomponente haben, die $1 / \sin \vartheta$ proportional ist, und hat damit in Bewegungsrichtung den Betrag

$$
K=\frac{\Delta v_{0}}{2 r_{\mathrm{L}}} \eta \sum \frac{\Delta F_{i}}{\sin ^{2} \vartheta_{i}},
$$

woraus folgt:

$$
\Delta v_{0}=\frac{K}{4 \pi r^{2} \eta} \frac{2 r_{\mathrm{L}}}{1 / \sin ^{2} \vartheta}
$$

Für $r=r_{\mathrm{L}}$ und dichteste Kugelpackung ist die (günstige) Translationsrichtung die, bei der für 6 Nachbarn $\sin \vartheta=1$, für die übrigen $6 \sin \vartheta=3^{-1 / 2}$ ist, also der Mittelwert:

$$
\overline{\frac{1}{\sin ^{2} \vartheta}}=\frac{1}{12}\left[6 \cdot 1^{2}+6 \cdot\left(3^{-1 / 2}\right)^{2}\right]=2 .
$$

Also ist für $r=r_{\mathrm{L}}$ :

$$
\Delta v_{0}=\frac{K}{4 \pi r \eta} \text {. }
$$

Vergleicht man dies mit Gl. (27), so sieht man, daß für die erste Schicht $c_{0}=1 / 2$ ist. Gegenüber dem Wert 2/3, der sich aus der ursprünglichen Ableitung ergab, macht dies keinen großen Unterschied. Man wird jedoch annehmen dürfen, daß für die erste Schicht in molekularen Bereichen derWert $c_{0}=1 / 2$ den Verhältnissen etwas besser gerecht wird. Dies bedeutet, daß in Gl. (24) im ersten Glied an Stelle des Faktors 2 der Wert 1,5 stehen muß. Wir erhalten also

$$
f_{\mathrm{t}}^{\prime}=\left(1,5 \frac{r_{\mathrm{L}}}{r}+\frac{1}{1+r_{\mathrm{s}} / r}\right)^{-1} .
$$

Für $r \approx r_{\mathrm{L}}$ läßt sich dies entwickeln in

$f_{\mathrm{t}}=0,5+0,31 \frac{r-r_{\mathrm{L}}}{r_{\mathrm{L}}}=0,19+0,31 \frac{r}{r_{\mathrm{L}}}$.

Dieser Wert sollte sich von dem wahren Wert $f_{\mathrm{t}}$ nur noch unterscheiden, wenn zwischen Lösungsmittel und gelöstem Teilchen andere Kräfte als im Lösungsmittel selbst herrschen, sowie durch Effekte etwaiger ,,selbständiger" Platzwechsel.

\section{Zur Diskussion der Experimente}

Die beiden letzten Abschnitte zeigten, daß die Berücksichtigung der Tatsache, daß die gleitenden Schichten eine endliche Dicke besitzen, eine wesentliche Reduktion der Mikroreibung ergibt. Die Reduktion ist verschieden für Translation und Rotation. Für gleichen Radius des Gelösten und der Lösungsmittelmoleküle, $r / r_{\mathrm{L}}=1$, ist der Mikroreibungsfaktor der Translation etwa 0,5, der der Rotation etwa 0,16; der Anstieg mit dem Radienverhältnis $r / r_{\mathrm{L}}$ ist annähernd linear. Unsicherheiten, die $20 \%$ nicht überschreiten dürften, könnten von Abweichungen von den idealisierten geometrischen Verhältnissen herrühren. Innerhalb dieser Grenzen jedoch sollten unsere Beziehungen die viskose Mikroreibung beschreiben.

Zum Vergleich mit den Experimenten werden in den Abb. 2 und 3 die theoretischen Mikroreibungs- 
faktoren mit den experimentellen aus der vorangehenden Arbeit ${ }^{1}$ verglichen. Die Größe und das leichte Anwachsen der Mikroreibung werden durch die Theorie ziemlich gut getroffen. Dies zeigt, daß die Berücksichtigung der Struktur beim Reibungsvorgang den Beobachtungen in den meisten Fällen gerecht wird. Ist im Einzelfall die Reibung größer,

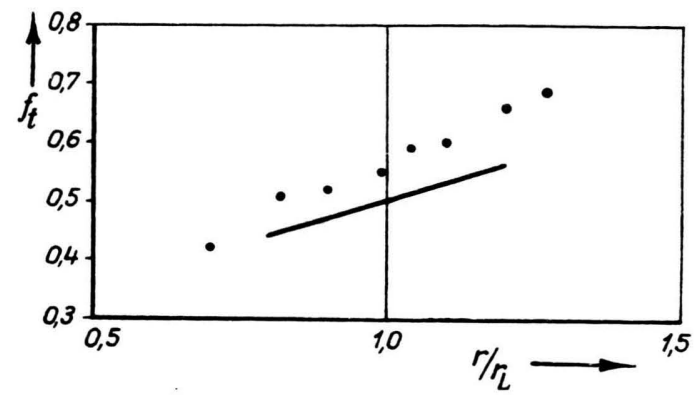

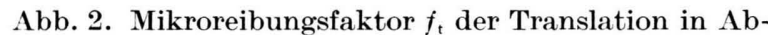
hängigkeit vom Radienverhältnis $r / r_{\mathrm{L}} ; r=$ Radius des wandernden Teilchens ; $r_{\mathrm{L}}=$ Radius der Lösungsmittelmoleküle. Punkte: Experimentelle Mittelwerte nach ${ }^{1}$ mit der Raumerfüllung $\varkappa=0,74$. Kurve: Theoretischer Wert nach Gl. (33).

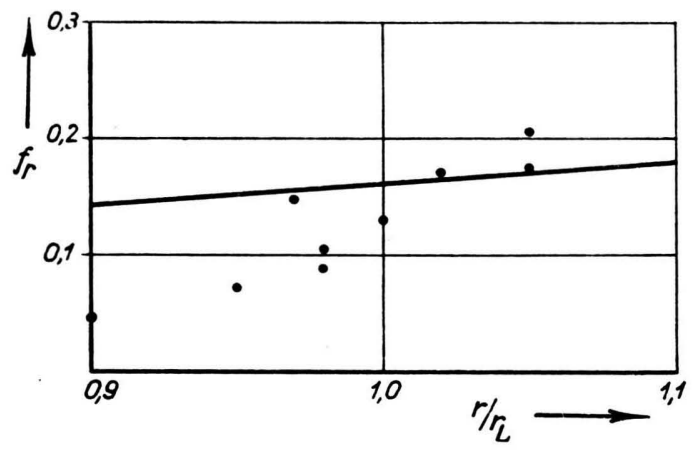

Abb. 3. Mikroreibungsfaktor $f_{\mathrm{r}}$ der Rotation in Abhängigkeit vom Radienverhältnis $r / r_{\mathrm{L}} ; r=$ Radius des rotierenden Teilchens; $r_{\mathrm{L}}=$ Radius der Lösungsmittelmoleküle. Punkte: Experimentelle Werte nach den Messungen von $W$ hiffe $n^{1,3}$ mit Raumerfüllung $\varkappa=0,74$. Kurve: Theoretischer Wert nach Gl. (21).

so darf demnach auf stärkere Kräfte zwischen Gelöstem und Lösungsmittel geschlossen werden, als zwischen den Lösungsmittelmolekülen selbst herrschen. Ein Grenzfall wäre eine feste Hydrathülle und eine entsprechende Vergrößerung des Radius. Auf eine sich darauf gründende Methode zur Bestimmung von Hydrathüllen kommen wir in anderem Zusammenhang zurück. In solchen Fällen sollten im

${ }^{3}$ D. H.Whiffen, Trans. Faraday Soc. 46, 124 [1950]. allgemeinen die Temperaturabhängigkeiten der Reibung und der Viskosität verschieden sein. Ein Beispiel bietet vielleicht die Diffusion verschiedener Substanzen in dem sehr wenig zähen Äthyläther (vgl. vorangehende Arbeit ${ }^{1}$, Tab. 1). Die Mikroreibung ist durchweg größer als man erwartet. Eine scheinbare Radienerhöhung infolge größerer Kräfte zwischen Gelöstem und Lösungsmittel würde dies erklären. Sind umgekehrt die Kräfte zwischen Gelöstem und Lösungsmittel kleiner als im Lösungsmittel selbst, so wird das Teilchen häufiger ,,selbständige" Platzwechsel ausführen können, die Mikroreibung wird also weiter herabgesetzt. Auch bei der Rotation muß mit selbständigen Platzwechseln gerechnet werden.

Die Gl. (33) stimmt nicht genau mit der empirisch für $f_{\mathrm{t}}$ [Gl. (9) der vorangehenden Arbeit $^{1}$ : $\left.f_{\mathrm{t}}=0,16+0,4 r / r_{\mathrm{L}}\right]$ überein, doch liegt die Abweichung, wie bereits in ${ }^{1}$ (siehe Diskussion der dortigen Abb. 6) bemerkt, völlig innerhalb der Meßfehler.

\section{Berücksichtigung selbständiger Platzwechsel}

$\mathrm{Zu}$ der Bewegung des Teilchens mögen ,selbständige Platzwechsel" im Sinne der Überlegungen des Abschnitts 2 beitragen. Dann kann man sich die resultierende Geschwindigkeit (der Translation bzw. Rotation) additiv zusammengesetzt denken aus dem Anteil $v_{1}$ des viskosen Mechanismus und dem Anteil $v_{2}$ der selbständigen Platzwechsel:

$$
v=v_{1}+v_{2} .
$$

Mit wachsendem Radienverhältnis dürfte $v_{2}$ verschwinden. Auch $v_{2}$ trägt deshalb zur Radienabhängigkeit des Mikroreibungsfaktors $f$ bei. Auch die Reibungskonstante $\varrho=K / v$ besteht entsprechend aus 2 Anteilen

$$
\frac{1}{\varrho}=\frac{1}{\varrho_{1}}+\frac{1}{\varrho_{2}}=\frac{1}{\varrho \text { Stokes } f^{\prime}}+\frac{1}{\varrho_{2}} ;
$$

$\varrho$ stokes steht für die klassischen Stokesschen Ausdrücke (3) und (4) und $f^{\prime}$ berücksichtigt den Struktureinfluß im Sinne der Abschnitte 3 und 4. Nach (5) erhält man hieraus den Mikroreibungsfaktor

$$
f=\frac{\varrho \text { mikro }}{\varrho \text { Stokes }}=\frac{1}{\frac{1}{f^{\prime}}+\frac{\varrho \text { Stokes }}{\varrho_{2}}}=\frac{f^{\prime}}{1+\frac{v_{2}}{v_{1}}} .
$$

Dieses ist der allgemeine Ausdruck für den Mikroreibungsfaktor. Es gilt für Translation und Rota- 
tion in gleicher Weise. Im Prinzip ermöglicht er, aus einer gemessenen Geschwindigkeit bzw. Reibung zusammen mit $f^{\prime}$ den Anteil des nichtviskosen Prozesses $v_{2}$ bzw. $\varrho_{2}$ abzuleiten.

Das Ziel künftiger experimenteller Untersuchungen sollte darin bestehen, an besonders niedrig- und hochviskosen Flüssigkeiten sowie an Hand der Temperaturabhängigkeit des Mikroreibungsfaktors $f$ die Brauchbarkeit unserer Überlegungen zu prüfen. Hinsichtlich der Rotation wäre eine Erweiterung experimentellen Materials auf größere Radienverhältnisse bei Teilchen ohne freie Drehgruppen besonders erwünscht.

Herrn A. Spernol und Herrn Prof. W. Heisenberg danken wir für wertvolle Hinweise.

\title{
Über den Einfluß äußerer Magnetfelder auf die Aktivität ferromagnetischer Katalysatoren
}

,Äußerer magnetokatalytischer Effekt" I

\author{
Von E. Justi und G. Vieth
}

Aus dem Institut für technische Physik der Technischen Hochschule Braunschweig (Z. Naturforschg. 8a, 538-546 [1953]; eingegangen am 25. Juli 1953)

Herrn Professor Dr. Walter Noddack zum 60. Geburtstag am 1\%. August 1953 in alter Freundschaft gewidmet

J. A. Hedvall has discovered "internal" magneto-catalytic effects; the activity of ferromagnetic catalyst changes upon transgressing the Curie-interval. The present publication deals with finding an "external" magneto-catalytic effect consisting of the influence of external magnetic fields upon the activity of ferromagnetic catalysts. This can be shown quite clearly in capillaries of high purity ferromagnetic nickel by experiments applying the $o$ - $p$-hydrogen conversion. In this reaction the conversion rises so steeply with relatively low field strengths that even the magnetic earth field should enhance the catalytic activity of nickel in compact form; a few hundred oersted may suffice for doubling the conversion and at some 1000 or 10.000 oersted a saturation value of the conversion is reached. The connection between catalytic activity and magnetism finds further support by a corresponding catalytic retentivity.

From the theory of ferromagnetism, especially the studies of Néel, it is known that extremely small ferromagnetic particles of the order of magnitude of Blochs walls, i. e. some $10^{-4} \mathrm{~mm}$, posses high permanent magnetism. This fact appears significant in this connection for the high catalytic activity of finely divided powder catalysts such as Raney catalysts. This permanent magnetism explains also the apparent lack of the external magneto-catalytic effect when using a nickel powder catalyst. The theoretical treatment of the results cannot yet give a definite explanation; before this can be given the experiments must be extended to ordinary chemical reactions. To this end the prerequisits for such experiments are being established.

Independently thereof the experimental proof for the external catalytic effect serves to ascertain that the internal Hedvall effects are primarily correlated to the ferromagnetic and paramagnetic states of matter.

\section{Problemstellung}

$\mathrm{I}^{\mathrm{s}}$ m Rahmen seiner vielfältigen Untersuchungen über nicht thermische Einflüsse auf die chemische Aktivität von Festkörpern entdeckte Hedvall ${ }^{1}$ einen magnetokatalytischen Effekt, der in Anomalien der katalytischen Aktivität ferromagnetischer Katalysatoren beim Durchschreiten des Curie-Intervalls besteht. Abb. 1 zeigt als Beispiel die Geschwindigkeit des $\mathrm{N}_{2} \mathrm{O}$-Zerfalles ${ }^{2}$ gemäß $2 \mathrm{~N}_{2} \mathrm{O} \rightarrow$ $2 \mathrm{~N}_{2}+\mathrm{O}_{2}$ über Ni als Funktion der Temperatur, wobei die Geschwindigkeitskurven im Curie-Be- reich $\left(\sim 360^{\circ} \mathrm{C}\right)$ von geringerer Neigung im ferromagnetischen Bereich zu größerer Steilheit im paramagnetischen Zustand des $\mathrm{Ni}$ übergehen. Noch aufschlußreicher ist die Kurve seines Mitarbeiters $\mathrm{Cohn}^{3}$ über den Zerfall der Ameisensäure in $\mathrm{H}_{2}$ und $\mathrm{CO}_{2}$ über $\mathrm{Co} / \mathrm{Pd}$-Legierung, denn diese Darstellung im $(\log k, 1 / T)$-Diagramm (Abb. 2) läßt erkennen,

1 J. A. Hedvall, Z. physik. Chem. 209, 445 [1935]. 2 J. A. Hedvall, R. Hedin u. O. Persson, Z. physik. Chem., Abt. B $\mathbf{2 7}, 196$ [1934].

${ }^{3}$ G. Cohn, Svensk kem. Tidskr. 52, 49 [1940]. 